\title{
Wolman Disease with a Low Cholesterol Level: An Unusual Laboratory Finding
}

Phawin Kor-anantakul, M.D. ${ }^{1}$, Thipwimol Tim-Aroon, M.D. ${ }^{2}$, Somchit Jaruratanasirikul, M.D. ${ }^{1}$

'Department of Pediatrics, Faculty of Medicine, Prince of Songkla University, Hat Yai, Songkhla 90110, Thailand.

2Department of Pediatrics, Faculty of Medicine, Ramathibodi Hospital, Mahidol University, Ratchatewi, Bangkok 10400, Thailand. Received 1 May $2020 \bullet$ Revised 12 November 2020 • Accepted 20 November $2020 \bullet$ Published online 16 April 2021

\section{Abstract:}

Wolman disease is a very rare autosomal recessive genetic disorder. The patients have the typical clinical finding of hepatosplenomegaly but with an abnormal lipid profile of high levels of total cholesterol (TC), triglycerides and lowdensity lipoprotein cholesterol (LDL-C), but a low level of high-density lipoprotein cholesterol (HDL-C). We report a 1-month-old boy with Wolman disease who had hepatosplenomegaly but with an atypical abnormal lipid profile of low TC level, and very low levels of both LDL-C and HDL-C. The genetic study revealed a compound heterozygous mutation of the LIPA gene, leading to the confirmed diagnosis of Wolman disease.

Keywords: LIPA gene, lysosomal acid lipase deficiency, Wolman disease

Contact: Prof. Somchit Jaruratanasirikul, M.D.

Department of Pediatrics, Faculty of Medicine, Prince of Songkla University, Hat Yai, Songkhla 90110, Thailand.

E-mail: somchit.j@psu.ac.th

M. Hosting by Prince of Songkla University. All rights reserved.

This is an open access article under the CC BY-NC-ND license

(http://www.jhsmr.org/index.php/jhsmr/about/editorialPolicies\#openAccessPolicy). 


\section{Introduction}

Wolman disease is a very rare autosomal recessive disorder with an estimated incidence of less than 1 in 100,000 live births. ${ }^{1}$ It is the most severe form of lysosomal acid lipase ( $L A L)$ deficiency, caused by a mutation of the LIPA gene located on chromosome 10q23.2-q23.3. ${ }^{1}$ This disorder was first described in 1961 by Wolman et al. in 3 siblings who had xanthomatous changes in several visceral organs. ${ }^{2}$ Since then, there have been about 70 Wolman cases reported worldwide, mostly from Middle Eastern and Caucasian countries. ${ }^{2-4}$ The only Asian country that has reported cases of Wolman disease is Japan, with 15 cases. ${ }^{5}$

Our report adds a 1-month-old boy from Thailand with Wolman disease who had a clinical presentation of severe hepatosplenomegaly with an abnormal lipid profile of low cholesterol and high triglyceride levels and very low levels of both low-density lipoprotein cholesterol (LDL-C) and high-density lipoprotein cholesterol (HDL-C). A genetic study revealed a compound heterozygous mutation of the LIPA gene.

\section{Case report}

A 1-month-old boy was referred for evaluation of progressive abdominal distension for 2 weeks. He was exclusively breastfed without any other gastrointestinal symptoms, no vomiting or constipation. He was born at term from a nonconsanguineous couple with birthweight of 2.7 kilograms $(\mathrm{kg})$. The physical examination found weight $4.5 \mathrm{~kg}$, marked abdominal distension from severe hepatosplenomegaly with the liver palpable 10 centimeters (cm) below the right costal margin and spleen $10 \mathrm{~cm}$ below the left costal margin. Blood chemistry showed hyponatremia, liver impairment and an abnormal lipid profile (sodium 119, potassium 4.6, chloride 89 and $\mathrm{CO}_{2}$ 19 millimoles per liter ( $\mathrm{mmol} / \mathrm{L})$, aspartate transferase 841, alanine transferase 110, alkaline phosphatase 297 international units per liter (IU/), total cholesterol (TC) 66, triglycerides (TG) 198, HDL-C 9.3, LDL-C 8.2 milligrams per deciliter (mg/dL), albumin 2.9, and globulin 3.6 grams per deciliter $(\mathrm{g} / \mathrm{dL})$.

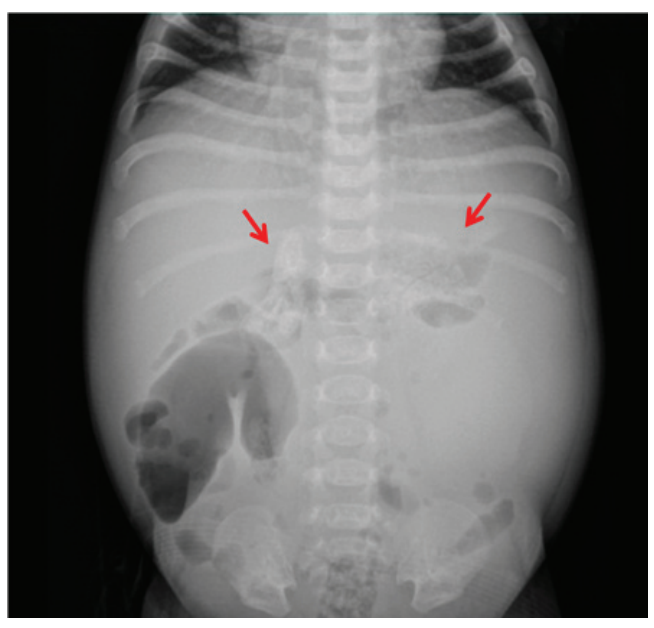

Figure 1 Plain film abdomen, note bilateral adrenal calcification

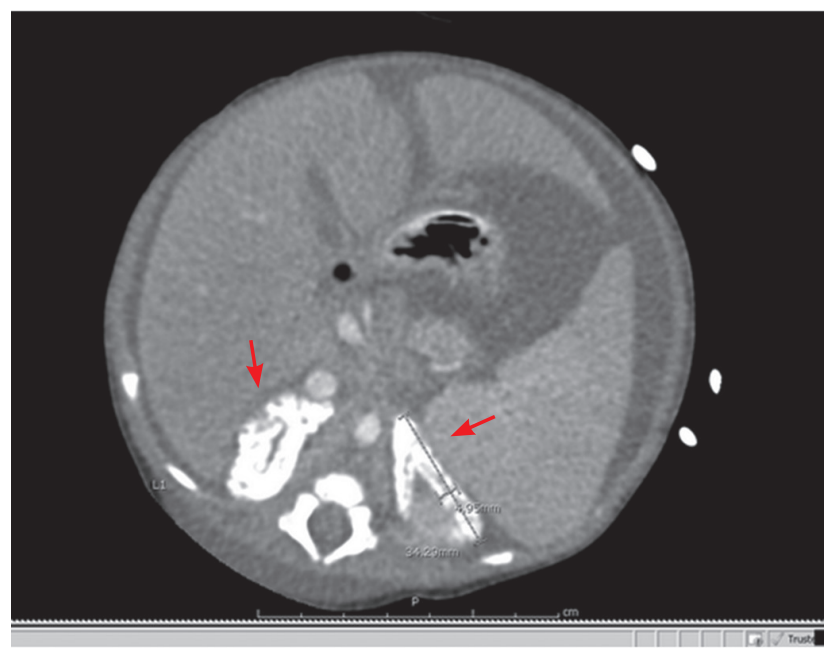

Figure 2 Computed tomography of the abdomen, note bilateral adrenal calcification

Investigations for an intrauterine infection were negative for toxoplasmosis, cytomegalovirus, rubella and herpes simplex viruses. A plain film of the abdomen showed calcification at both adrenal glands (Figure 1), which was confirmed by computed tomography (Figure 2). A dried 
blood spot on filter paper was sent to the National Taiwan University Hospital to test for lysosomal acid lipase activity
(4MU-fluorometric assay) which found a very low level of 0.007 nmoles per punch per hour (reference range $>0.13$ ).

\begin{abstract}
p.A141Vfs X144 in exon4 of LIPA gene, a heterozygous 8-base pair deletion (GCTTTCAG) of coding nucleotide 421-428, causing the amino acid change at codon 141 from alanine to valine followed by reading frameshift and premature termination at codon 144 . The father of the patient
\end{abstract} was identified to be a carrier of heterozygous p.A141Vfs X144.

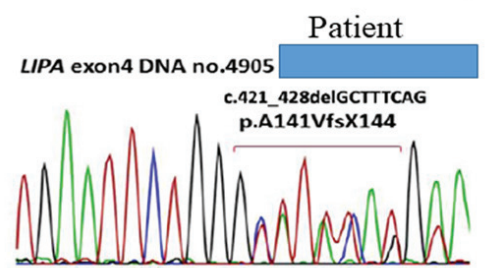

Patient's father

LIPA exon4 DNA no.4906

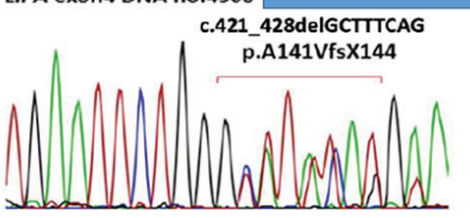

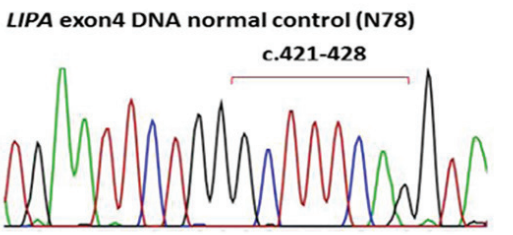

Patient's mother

LIPA exon4 DNA no.4907

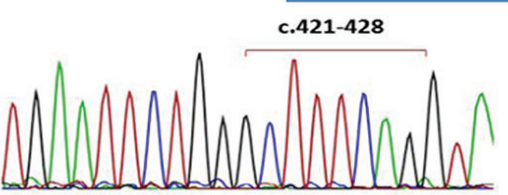

A

p.E232X in exon7 of LIPA gene, a heterozygous nucleotide no.694 change from $\mathrm{G}$ to $\mathrm{T}$ causir the amino acid no.232 change from glutamine to stop codon (GAA>TAA). The mother of the patient was identified to be a carrier of heterozygous p.E232X.

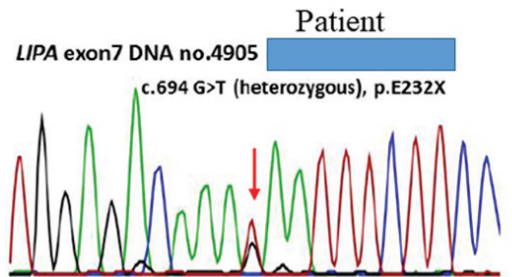

Patient's father

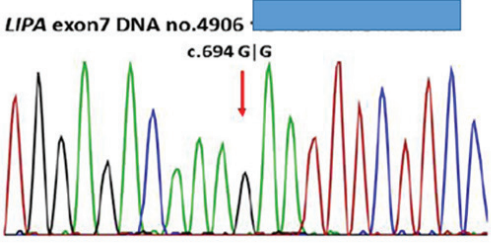

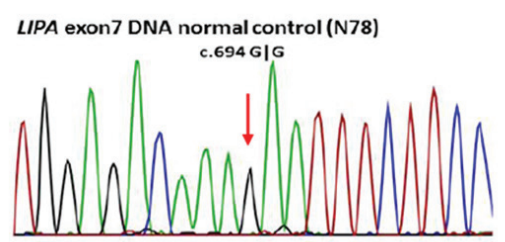

Patient's mother

LIPA exon7 DNA no.4907

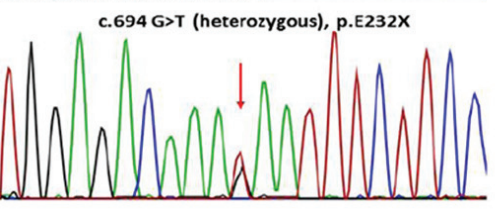

B

Figure 3 Genetic studies

(A) Heterozygous mutation of the LIPA gene, exon 4

(B) Heterozygous mutation of the LIPA gene, exon 7 
Genomic deoxyribonucleic acid (DNA) was extracted from the patient's and his parents' peripheral blood following standard protocols (QIAGEN GmbH). Polymerase chain reaction amplification and direct sequencing of all coding exons (exon 2-10) of the LIPA gene (NM_000235.4) were performed. Further genetic study demonstrated a compound heterozygous mutation of the LIPA gene of an 8-bp deletion (c.421_428delGCTTTCAG, p.A141VfsX144.) at a donor splice site on exon 4, and a heterozygous nonsense mutation at exon 7 (c.694G>T, p.E232X). Both variants were novel, and classified as pathogenic variants. Genetic studies of the parents revealed that the father was a heterozygous carrier for a frameshift mutation, p. A141VfsX144, and the mother was a heterozygous carrie for the nonsense mutation, p.E232X (Figure 3).

The patient was treated with empirical antibiotics, oxygen therapy and parenteral nutritional support. After discussion with his parents concerning the lethal prognosis of Wolman disease and the optional therapy of stem cell transplantation, the parents decided on palliative treatment. The patient expired after hospitalization for 3 weeks. The parents were informed of the $25.0 \%$ recurrence risk in the next pregnancy and prenatal diagnosis was recommended.

\section{Discussion}

The patient was a 1-month-old boy referred to our hospital for evaluation of severe anemia and marked hepatosplenomegaly in which the initial differential diagnosis was intrauterine infection or hematologic malignancy. The presence of bilateral adrenal calcification revealed on a plain abdominal film led the clinician to suspect a diagnosis of Wolman disease. A very low level of lysosomal acid lipase activity confirmed the $\mathrm{LAL}$ deficiency. The genetic study findings of a compound heterozygous mutation of the LIPA gene in the patient of an 8-bp deletion (c.421_428delGCTTTCAG, p. A141VfsX144.) at a donor splice site on exon 4, and a heterozygous nonsense mutation at exon 7 (c.694G>T, p.E232X) confirmed the diagnosis of Wolman disease. Both father and mother were heterozygous carriers as the father was found to be a carrier of a frameshift mutation, p. A141VfsX144 and the mother was a carrier of a nonsense mutation, p.E232X.

Wolman disease is a lysosomal storage disease caused by an LAL deficiency, and an inherited autosomal recessive genetic disease caused by a mutation of the LIPA gene. The LAL enzyme plays an important role in breaking down certain fatty materials (cholesterol esters and triglycerides) in all cells except red blood cells, which have no lysosomes. Lack of this enzyme leads to intracellular accumulation of cholesterol esters and triglycerides in all body organs resulting in progressive organomegaly with impaired function. ${ }^{6}$ The serum lipid profile typically shows high levels of TC, TG and LDL-C but a low level of HDL-C from impaired lipid degradation secondarily to lack of the LPL enzyme. The accumulation of lipids in intestinal mucosa leads to poor macro- and micronutrient absorption resulting in malnutrition and failure to thrive.

Since the disease was first reported in 1961, there have been about 70 cases worldwide with the largest case series from western countries as reviewed by Aguisanda et al. ${ }^{3}$ and Jones et al. ${ }^{4}$, and one series from Japan reviewed by Ikari et al. ${ }^{5}$ The median age at diagnosis of these cases was 2.5 months with presenting symptoms of growth failure, malnutrition and hepatosplenomegaly, with abnormal serum lipid profiles of high levels of TC (average $115 \mathrm{mg} /$ $\mathrm{dL}$ ), TG (average $215 \mathrm{mg} / \mathrm{dL})^{4}$, and high LDL-C, but a low level of $\mathrm{HDL}-\mathrm{C}^{2-4}$

In our patient, the serum TG level was very high at $198 \mathrm{mg} / \mathrm{dL}$ indicating impaired lipid degradation from lack of the $L A L$ enzyme. Somewhat strangely, the levels of TC, LDL-C and HDL-C were all low, which is not typical in Wolman disease. The unusually low cholesterol levels in our patient could have been due to various reasons. First, our patient developed hepatomegaly during the first 
month of life, indicating severe organ involvement and impaired liver function (shown by the low level of serum albumin) beginning shortly after birth. As the major source of circulating cholesterol is from endogenous synthesis in the liver, our patient might have had impaired endogenous cholesterol synthesis resulting in a low level of TC. Second, severe intracellular lipid accumulation in the intestinal wall could have resulted in poor intestinal lipid absorption. Another possible explanation of the very low levels of TC and LDL-C in our patient could be that he was exclusively breastfed. It is known that the average lipid content in mature human milk is $3.5-4.5 \mathrm{~g} / 100 \mathrm{~mL}$ with the major component of triglycerides at $98.0-99.0 \%$, and a small amount of cholesterol of $0.2-0.3 \%{ }^{7,8}$ However, some other previous infant cases with Wolman disease were also exclusively breastfed, but they still had high levels of TC and LDL-C. ${ }^{3-5}$

Wolman disease is a fatal disorder. The majority of affected patients die within 6-12 months with the median age at death of 3.5 months from malnutrition and multiorgan failure. ${ }^{3-5}$ Hematopoietic stem cell transplantation has been tried to treat this disease, but with unfavorable outcomes in some patients. ${ }^{9}$ In 2015, sebelipase alfa, a recombinant form of human LAL, was approved in the United States of America, European Union and Japan for long term enzyme replacement therapy in patients with $L A L$ deficiency with patients being given this drug showing clinical improvement of weight gain and reduced abdominal distension from decreased hepatosplenomegaly..$^{10-12}$

In summary, we describe the case of a 1-month-old patient who presented with progressive abdominal distension from hepatosplenomegaly and an abnormal lipid profile featuring low levels of TC, LDL-C and HDL-C. Wolman disease was suspected due to the presence of bilateral adrenal calcification. The diagnosis was confirmed by a genetic study which revealed a compound heterozygous mutation of the LIPA gene.

\section{Conclusion}

Wolman disease is a very rare autosomal recessive genetic disorder. We report a 1-month-old patient with typical clinical findings of hepatosplenomegaly but with an atypical abnormal lipid profile including a low TC level and very low levels of both LDL-C and HDL-C. The genetic study revealed a compound heterozygous mutation of the LIPA gene.

\section{Acknowledgement}

The authors thank Mr. David Patterson from the International Affairs Office of the Faculty of Medicine, Prince of Songkla University, for English editing of the manuscript.

\section{Funding sources}

None

\section{Conflict of interest}

None

\section{References}

1. Hamosh A. Lysosomal acid lipase deficiency \# 278000 [homepage on the Internet]. Baltimore: Johns Hopkins University; 2018 [cited 2020 Sep 14]. Available from: https:// www.omim.org/entry/278000

2. Wolman M, Sterk VV, Gatt S, Frenkel M. Primary familial xanthomatosis with involvement and calcification of the adrenals: report of two more cases in siblings of a previously described infant. Pediatrics 1961;28:742-57.

3. Aguisanda F, Thorne N, Zheng W. Targeting Wolman disease and cholesteryl ester storage disease: disease pathogenesis and therapeutic development. Curr Chem Genom Transl Med 2017; $11: 1-18$.

4. Jones SA, Valayannopoulos V, Schneider E, Eckert S, Banikazemi M, Bialer M, et al. Rapid progression and mortality of lysosomal acid lipase deficiency presenting in infants. Genet Med 2016;18: 452-8.

5. Ikari N, Shimizu A, Asano T. Lysosomal acid lipase deficiency in Japan: a case report of siblings and a literature review of cases in Japan. J Nippon Med Sch 2018;85:131-7. 
6. Reiner Ž, Guardamagna O, Nair D, Soran H, Hovingh K, Bertolini $\mathrm{S}$, et al. Lysosomal acid lipase deficiency - an under-recognized cause of dyslipidaemia and liver dysfunction. Atherosclerosis 2014;235:21-30.

7. Lindquist $\mathrm{S}$, Hernell $\mathrm{O}$. Lipid digestion and absorption in early life: an update. Curr Opin Clin Nutr Metab Care 2010;13:314-20.

8. Delplanque B, Gibson R, Koletzko B, Lapillonne A, Strandvik B. Lipid quality in infant nutrition: current knowledge and future opportunities. J Pediatr Gastroenterol Nutr 2015;61:8-17.

9. Yanir A, Allatif MA, Weintraub M, Stepensky P. Unfavorable outcome of hematopoietic stem cell transplantation in two siblings with Wolman disease due to graft failure and hepatic complications. Mol Genet Metab 2013;109:224-6.

10. Shirley M. Sebelipase alfa: first global approval. Drugs 2015; 75:1935-40.

11. Frampton JE. Sebelipase alfa: a review in lysosomal acid lipase deficiency. Am J Cardiovasc Drugs 2016;16:461-8.

12. Jones SA, Rojas-Caro S, Quinn AG, Friedman M, Marulkar S, Ezgu $F$, et al. Survival in infants treated with sebelipase alfa for lysosomal acid lipase deficiency: an open-label, multicenter, dose-escalation study. Orphanet J Rare Dis 2017;12:25. 